\title{
3 Research Square \\ The Efficacy of Strokectomy in the Treatment of Malignant Cerebral Infarction Patients
}

\section{Ning Li}

Jinan University First Affiliated Hospital

Chunmei He

Guangdong 999 Brain Hospital

Wei Li

Jinan University First Affiliated Hospital

Liu Zhang

Jinan University First Affiliated Hospital

Yehai Li

Guangdong 999 Brain Hospital

\section{Yongjian Feng}

Jinan University First Affiliated Hospital

Weilong Ding

Jinan University First Affiliated Hospital

Min Guan

Jinan University First Affiliated Hospital

Xiangyu Wang

Jinan University First Affiliated Hospital

Shiyong Wang ( $\sim 18127968231 @ y e a h . n e t)$

Jinan University First Affiliated Hospital

\section{Research}

Keywords: strokectomy, decompressive hemicraniectomy, malignant cerebral infarction, brain herniation, intracranial pressure

Posted Date: January 25th, 2021

DOI: https://doi.org/10.21203/rs.3.rs-151694/v1

License: (c) (i) This work is licensed under a Creative Commons Attribution 4.0 International License. Read Full License 


\section{Abstract}

\section{Introduction}

Strokectomy refers to the resection of infarct brain tissue. Used alone, in combination with decompressive hemicraniectomy (DHC), or as a remedial surgery to $\mathrm{DHC}$ for malignant cerebral infarction $(\mathrm{MCl})$ patients, strokectomy has reduced mortality rates and improved functional outcomes of patients with $\mathrm{MCl}$. However, the role of strokectomy in the treatment of $\mathrm{MCl}$ patients is controversial. The aim of this retrospective study was to investigate the efficacy of strokectomy in $\mathrm{MCl}$ treatment in order to explore its beneficial effects on improving patient outcomes.

\section{Methods}

This retrospective study was carried out between January 2017 and September 2019 in the First Affiliated Hospital of Jinan University and Guangdong 999 Brain Hospital in Guangzhou, China. We reviewed patients with $\mathrm{MCl}$ who underwent $\mathrm{DHC}$ with or without strokectomy. We collected and analyzed the following data for all patients: demographics, Glasgow Coma Scale scores (GCS), National Institutes of Health Stroke Scale (NIHSS) scores, observational data in the intensive care unit (ICU), post-surgery intracranial pressure (ICP) monitoring, midline shift before and after surgery, and functional outcomes measured with the modified Rankin Scale (mRS) at 6 months.

\section{Results}

We recruited 95 patients (53 men; mean age, $59.71 \pm 10.65$ years; age range, 38 - 78 years). After surgery, patients who received DHC and strokectomy were associated with a lower ICP curve; decreased midline shift; and less mannitol, hypothermia, and hypertonic saline therapies than those who received DHC alone. No patient in the $\mathrm{DHC}+$ strokectomy group had malignant high ICP or needed remedial surgery; in contrast, 16 patients in the DHC group had malignant high ICP after DHC, and remedial surgery was recommended. Five patients received remedial surgery and survived, while the 11 who refused remedial surgery died. The mortality rate during ICU stay was $19.4 \%$ in the DHC group and $7.1 \%$ in the DHC+strokectomy group. While the rate of poor outcomes and mortality was significantly different between the two groups at 6 months after surgery, good outcomes did not differ significantly.

\section{Conclusions}

Strokectomy performed in combination with DHC can effectively decrease post-surgery ICP, reduce midline shift, and reduce mortality during the six months following surgery. Moreover, strokectomy performed as a remedial surgery could decrease malignant high ICP after DHC and reduce mortality.

\section{Introduction}

The introduction of decompressive hemicraniectomy (DHC) as a treatment for malignant cerebral infarction $(\mathrm{MCl})$ has reduced the mortality rate of $\mathrm{MCl}$ from approximately $80 \%$ to $20-40 \%$ [1][2][3][4][5]. 
Although the decrease in mortality is a great advancement in the treatment of $\mathrm{MCl}$ patients, it is still high enough to warrant further study. The primary reason for the relatively high mortality after DHC may be that even undergoing maximal $\mathrm{DHC}$, some patients with $\mathrm{MCl}$ experience progressive swelling of the affected hemisphere and inevitable herniation that could be fatal for $\mathrm{MCl}$ patients [6][7][8][9][10]. Strokectomy, resection of the infarct brain tissue, used alone or in combination with DHC or as remedial surgery to $\mathrm{DHC}$, leads to lower mortality rates compared to patients treated with $\mathrm{DHC}$ only [8][10][11]. However, due to its intrinsic drawbacks, (e.g., more time needed to operate, higher blood loss, the possibility of removal of viable neural tissue), it remains a controversial option in the treatment of $\mathrm{MCl}$ patients [12].

Until now, there are no guidelines concerning the selection of treatment modalities for patients with $\mathrm{MCl}$; patients suitable for surgery are treated differently according to the preference of neurosurgeons. Selecting appropriate treatment modalities for patients with $\mathrm{MCl}$ remains to be elucidated. Therefore, the aim of this study was to investigate the efficacy of strokectomy used in combination with DHC, or as a remedial surgery to $\mathrm{DHC}$ in order to explore its beneficial effects in $\mathrm{MCl}$ treatment to improve patient outcomes.

\section{Methods}

\section{Patient selection}

We performed a retrospective study of patients with $\mathrm{MCl}$ who underwent decompressive hemicraniectomy with or without strokectomy between January 2017 and September 2019 in The First Affiliated Hospital of Jinan University (52 patients) and Guangdong 999 Brain Hospital (43 patients) in Guangzhou, China. Inclusion criteria were as follow: patients diagnosed with middle cerebral artery (MCA) infarction with or without ipsilateral anterior cerebral artery (ACA) or posterior cerebral artery (PCA) infarction and who showed severe swelling with compression of the ipsilateral lateral ventricle or midline shift on CT scans. Exclusion criteria were any of the following: patients with bilateral dilated pupils, severe comorbidity, reduced life expectancy, or bilateral large territory infarction. A total of 95 patients were included in this study. All subjects had written informed consent given by their caregivers and the study protocol was approved by the corresponding institute committee on human research.

\section{Procedure}

The surgical removal of a large bone flap was conducted as described by Subramaniam [13]. During the operation, some patients with edematous brain bulging after durotomy then underwent strokectomy. The extent of resection included the anterior temporal lobe and a portion of the frontal lobe above the Sylvian fissure. The fissure vein was protected with caution during the procedure. Intracranial pressure (ICP) was monitored with the intraparenchymal insertion of an ICP probe (Sophysa, France).

\section{Post-operative intensive care unit stay}


Immediately after surgery, patients were transferred under anesthesia, intubation, and mechanical ventilation to the neurosurgery intensive care unit (ICU), where they received the following therapy: antihypertensive medication, dehydrating agents (mannitol or hypertonic saline) if necessary, sedation, low-molecular-weight heparin, intravenous fluids, and proton-pump inhibitors. Mannitol therapy was administered when ICP persistently exceeded $15 \mathrm{~mm} \mathrm{Hg}$. Hypothermia was considered as an alternative therapy to decrease high ICP if malignant brain edema could not be alleviated with mannitol or hypertonic saline. Brain computed tomography (CT) scans were performed as needed to evaluate the patient's intracranial condition, and treatment strategies were adjusted accordingly. In cases of refractory elevated ICP, therapy was further enhanced with the repeated administration of mannitol injections, hypertonic saline, and hypothermia therapy if necessary. The persistence of refractory increasing ICP and midline shift accompanied by decreasing Glasgow Coma Scores (GCS) and pupil mydriasis despite the comprehensive multimodality treatment indicated the remove of the infarct tissue with remedial surgery. Permission and signed written consent was requested of the patients' caregivers to perform remedial strokectomy to alleviate the deadly mass-effect and decrease the high ICP. Conservative management was continued in case of refusal.

The appropriate timing of mechanical ventilation discontinuation and extubation depended on consciousness recovery and the severity of complications. If patients remained comatose for more than 7 days, tracheotomies were performed. The mechanical ventilation time and intubation time were recorded. ICP was monitored continuously until the peak stage of brain swelling had passed, and ICP began to decrease.

Patients were transferred to rehabilitation wards after meeting the following conditions: stable vital signs, no signs of herniation, no fever, controlled pulmonary infection, extubation or tracheotomies performed, maintenance of oxyhemoglobin saturation at normal levels without mechanical ventilation.

\section{Data Collection}

Clinical data were collected for all patients, including age, sex, baseline medical comorbidities, presence of pupillary asymmetry, NIHSS score, and GCS. All preoperative and postoperative brain CT scans and magnetic resonance images were reviewed to measure the midline shift at the level of the septum pellucidum before and after surgery. Duration of stay in ICU and the time needed for mechanical ventilation were recorded. Hypertonic saline therapy and refractory hypernatremia were also recorded. Mortality in ICU stay was recorded. Mortality and modified Rankin Scale (mRS) scores at 6 months after surgery were used as outcome parameters.

\section{Statistical analyses}

Statistical analyses were performed with SPSS 13.0 for Windows (SPSS Inc., Chicago, IL). P-values of less than 0.05 were considered statistically significant. Two-tailed Student $t$-tests were used for normally distributed variables. Mann-Whitney $\mathrm{U}$ tests were used for ordinal variables. Dichotomous variables were 
compared with Pearson's $\chi^{2}$ test or the Fisher's exact test when appropriate. ICP curves were generated with GraphPad Prism software (version 5.0).

\section{Results}

\section{Overview of the included patients}

The present retrospective study included 95 patients: 67 patients in the DHC group and 28 patients in the $\mathrm{DHC}+$ strokectomy $(\mathrm{DHC}+\mathrm{S}$ ) group (Fig. $1 \mathrm{~A}$ ). All patients were transferred to the ICU after surgery and to rehabilitation wards when vital signs were stable. The overall death rate at six months after surgery was 20\% (19/95): $14.7 \%$ (14/95) died during their ICU stay and 5.3\% (5/95) in the rehabilitation stage.

In the DHC group, 51 of 67 patients had normal or moderately increased intracranial pressure (NICP). Of the 51 patients, 49 became stable after treatment and were transferred to the rehabilitation ward, and two died (one due to a pulmonary embolism and one due to severe post-surgery intracranial infection). Of the 67 patients, 16 suffered from malignant high intracranial pressure (MICP) accompanied by decreasing GCS scores, increased midline shift and pupil mydriasis. Of these 16 patients, five underwent remedial surgery and survived after prolonged stays in the ICU; however, 11 refused remedial surgery and died from complications related to severe brain herniation. Of the 54 patients transferred to rehabilitation wards in the DHC group, four died (two of severe pneumonia and two of pulmonary embolism) in the following 6 months.

No patients in the DHC+S group developed malignant ICP during their stay in the ICU. One death in the ICU was caused by acute heart failure. In the rehabilitation stage, one patient died due to severe lung infection caused by aspiration.

\section{Preoperative data}

The preoperative data obtained from all the patients are presented in Table 1. Site of infarction and the severity of swelling measured with preoperative head CT differed significantly between the two treatment groups. Sex, age, preoperative GCS, preoperative NIHSS score, time from ictus to operation, and etiology of ischemia did not differ significantly between the treatment groups.

\section{Treatments in the ICU}

There were significant differences in the variables of mannitol therapy, hypothermia therapy, hypertonic saline therapy, remedial surgery recommended, and time of ICU stay between the two groups. No statistical differences in the variables of tracheotomy, acute renal failure, duration of mechanical ventilation, and mortality in ICU were observed between the DHC and DHC+S groups (Table 2). Refractory high ICP and increasing midline shift accompanied by decreasing GCS scores and pupil mydriasis were observed after surgery in $16 / 67$ patients in the DHC group $(23.89 \%)$ and in none of the patients in the $\mathrm{DHC}+\mathrm{S}$ group; hence, no patient in the latter group required further surgery. 


\section{Characteristics of patients in DHC group with or without malignant high ICP}

We observed 16 patients with refractory elevated ICP accompanied by decreasing GCS scores and pupil mydriasis that could not be alleviated with repeated mannitol injections, hypertonic saline, or hypothermia therapy. Remedial surgery to remove infarct tissue was proposed. We divided these patients into two groups for analysis (Table 3). Large territory infarctions and mannitol therapy were significantly more common among the 16 patients who were recommended to receive remedial surgery (group 1) than among the remaining 51 patients in the DHC group without malignant high ICP (group 2).

\section{Post-surgery ICP monitoring}

All patients were implanted with ICP probe to allow for the monitoring of their ICP (Fig. $1 \mathrm{~B}$ ). The calculation of ICP curves was based on the mean ICP of each patient per day in both groups. The data of five patients in the DHC group who underwent remedial surgery obtained on the day of the secondary surgery and afterwards were excluded from the calculation of ICP curves. Results are shown in Figure 1. The patients in the DHC+S group had lower ICP measurements.

\section{Midline shift calculations before and after surgery}

All patients were monitored with dynamic head CT scans following surgery. The midline shifts of these patients at the level of septum pellucidum were measured and compared. The results revealed that midline shifts decreased significantly after surgery in the DHC+S group, while those in the DHC group increased slightly, but not significantly, after surgery (Table 4).

\section{Functional Outcomes at 6 months}

The mRS was used as an outcome parameter to evaluate the quality of life of the patients at 6 months following surgery. The values of mRS obtained at 6 months were grouped and categorized as good outcomes (mRS 1-3), poor outcomes (mRS 4-5), and death (mRS 6). The distribution of $m R S$ is shown in Figure 1C. While the rate of poor outcomes or death differed significantly between the two groups, rates of good outcomes did not. In the DHC group, $29.9 \%$ of the patients had good outcomes at 6 months, $44.8 \%$ had poor outcomes, and $25.4 \%$ died; in the DHC+S group, $21.4 \%$ of the patients had good outcomes, $71.4 \%$ had poor outcomes, and $7.1 \%$ died.

\section{Discussion}

Previous randomized controlled trials (RCTs) (DESTINY, DECIMAL and HAMLET) have shown that DHC reduces mortality and improves functional outcomes of patients with $\mathrm{MCl}$ [14][15][16]. However, the mortality rate following $\mathrm{DHC}$ ranges between 20 and 40\% [4][17][18]. This high mortality is attributable to increasingly high ICP and herniation due to refractory brain swelling [7][19][20]. Patients with refractory increasing high ICP and midline shift accompanied by decreasing GCS scores and pupil mydriasis must elect to continue conservative treatment or receive remedial surgery. Concerning the latter option, the application of strokectomy as a remedial strategy has yielded favorable results [6][19][20]. 
Indicating that strokectomy used in combination with $\mathrm{DHC}$ or as remedial surgery to $\mathrm{DHC}$ can effectively reduce post-surgery ICP and decrease rates of mortality, our findings have the following implications: (1) as demonstrated by the decreased ICP, midline shift, and brain stem compression in the DHC+strokectomy group following surgery, strokectomy is effective in treating high post-surgery ICP; (2) strokectomy reduces mortality both when performed in combination with $\mathrm{DHC}$ or as a remedial surgery for patients with high ICP after DHC; (3) reduced mortality in DHCstrokectomy group resulted in an increased proportion of patients with poor outcomes; and (4) strokectomy is safe for and beneficial to $\mathrm{MCl}$ patients.

While the present and previous findings clearly indicate that strokectomy is a life-saving procedure for patients with malignant high ICP, strokectomy has remained controversial. For example, Wartenberg et al., Pandhi et al., and Cheung et al. have all advised against the resection of the infarction and strokectomy, as the margins between infarct and the penumbra are poorly defined, the procedure risks the removal of potentially viable neural tissue from the margins of the ischemic penumbra [21][22][23]. By contrast, Kostov et al. suggested that strokectomy alone may be equivalent to a DHC with or without brain resection. Since high ICP before or after DHC would further reduce perfusion of the remaining viable tissue, the removal of the anterior temporal lobe and uncus can directly relieve brainstem compression and rescue the penumbra, and the bone flap may be immediately replaced if sufficient strokectomy was achieved[11].

When strokectomy is indicated, the surgeon should ensure that sufficient infarct resection has been achieved. Insufficient strokectomy could prevent the attenuation of the high ICP and brain herniation. In contrast, viable tissue may most likely be removed with radical resection. While Kostov et al. suggested the use of indocyanine green-video angiography to guide the extent of resection, implementing this strategy during surgery would be excessively time-consuming[11]. We recommend the partial resection of the infarct: that of the anterior temporal lobe can prevent uncal herniation and brain stem compression, and the resection of a portion of the frontal lobe above the Sylvian fissure can avoid compression of the structure of the diencephalon.

Our research indicates that $14 / 16$ (87.5\%) patients with progressive swelling in the DHC group featured infarcts in regions beyond MCA territory that involved the ACA or PCA territories. This indicates that patients with large infarcts might develop malignant high ICP after DHC. ICP and midline shift could progressively deteriorate patient prognoses and elevate mortality rates up to $30-70 \%$. The realization of a second surgery, including strokectomy, may help decrease mortality rates and achieve better outcomes, especially in cases of infarction that involve more than one vascular territory.

\section{Conclusions}

Resection of infarct brain tissue, performed in combination with DHC or as a remedial surgery after DHC, is an effective and safe treatment of $\mathrm{MCl}$ that can significantly decrease post-surgery ICP and reduce mortality. However, due to the retrospective nature of this study, the operative procedures and the 
treatment in ICU were not standardized; hence, randomized, prospective study is warranted to confirm our conclusions.

\section{Abbreviations}

ACA: anterior cerebral artery; CT: computed tomography; DHC: decompressive hemicraniectomy; GCS: Glasgow Coma Scale scores; ICP: intracranial pressure; ICU: intensive care unit; MCA: middle cerebral artery; $\mathrm{MCl}$ : malignant cerebral infarction; MICP: malignant high intracranial pressure; mRS: modified Rankin Scale; NICP: moderately increased intracranial pressure; NIHSS: National Institutes of Health Stroke Scale; PCA: posterior cerebral artery; RCTs: randomized controlled trials.

\section{Declarations}

\section{Ethical Approval and Consent to participate}

All subjects gave written informed consent by their caregivers and the study protocol was approved by the Jinan University Ethics committee. (No. KY-2020-043)

\section{Consent for publication}

Not applicable.

\section{Availability of supporting data}

All data generated or analyzed during this study are included in this published article.

\section{Competing interests}

The authors declare that there are no competing interests.

\section{Funding}

Not applicable.

\section{Authors' contributions}

Ning Li, Chunmei He, Shiyong Wang and Xiangyu Wang contributed to patients enrollment, literature search, study design, data analysis, data interpretation, and writing of the manuscript.

Dr. Wei Li and Yehai Li contributed to data analysis, data interpretation, and critical review of the manuscript.

Dr. Liu Zhang, Yongjian Feng, Weilong Ding, Min Guan contributed to patient enrollment, data collection, and critical review of the manuscript. 
Acknowledgements

Not applicable.

\section{References}

1. Foerch C, Lang JM, Krause J, Raabe A, Sitzer M, Seifert V, et al. Functional impairment, disability, and quality of life outcome after decompressive hemicraniectomy in malignant middle cerebral artery infarction. J Neurosurg. 2004;101:248-54.

2. Unterberg A, Juettler E. The role of surgery in ischemic stroke: decompressive surgery. Curr Opin Crit Care. 2007;13:175-9.

3. Huttner HB, Schwab S. Malignant middle cerebral artery infarction: clinical characteristics, treatment strategies, and future perspectives. Lancet Neurol. 2009;8:949-58.

4. Gul W, Fuller HR, Wright H, Sen J. A Systematic Review and Meta-Analysis of the Effectiveness of Surgical Decompression in Treating Patients with Malignant Middle Cerebral Artery Infarction. World Neurosurg. 2018;120:e902-20.

5. Beez T, Munoz-Bendix C, Steiger H-J, Beseoglu K. Decompressive craniectomy for acute ischemic stroke. Crit Care. 2019;23:209.

6. Merenda A, Perez-Barcena J, Frontera G, Benveniste RJ. Predictors of clinical failure of decompressive hemicraniectomy for malignant hemispheric infarction. J Neurol Sci. 2015;355:54-8.

7. Paldor I, Rosenthal G, Cohen JE, Leker R, Harnof S, Shoshan Y, et al. Intracranial pressure monitoring following decompressive hemicraniectomy for malignant cerebral infarction. J Clin Neurosci. 2015;22:79-82.

8. Hinduja A, Samant R, Feng D, Hannawi Y. Herniation despite Decompressive Hemicraniectomy in Large Hemispherical Ischemic Strokes. J Stroke Cerebrovasc Dis. 2018;27:418-24.

9. Tartara F, Bongetta D, Colombo EV, Bortolotti C, Cenzato M, Giombelli E, et al. Strokectomy and Extensive Cerebrospinal Fluid Drainage for the Treatment of Space-Occupying Cerebellar Ischemic Stroke. World Neurosurg. 2018;115:e80-4.

10. Schwake M, Schipmann S, Müther M, Stögbauer L, Hanning U, Sporns PB, et al. Second-look strokectomy of cerebral infarction areas in patients with severe herniation. J Neurosurg. 2019;132:19.

11. Kostov DB, Singleton RH, Panczykowski D, Kanaan HA, Horowitz MB, Jovin T, et al. Decompressive hemicraniectomy, strokectomy, or both in the treatment of malignant middle cerebral artery syndrome. World Neurosurg. 2012;78:480-6.

12. Neugebauer $H$, Jüttler $E$. Hemicraniectomy for malignant middle cerebral artery infarction: current status and future directions. Int J Stroke. 2014;9:460-7.

13. Subramaniam S, Hill MD. Decompressive hemicraniectomy for malignant middle cerebral artery infarction: an update. Neurologist. 2009;15:178-84. 
14. Jüttler E, Schwab S, Schmiedek P, Unterberg A, Hennerici M, Woitzik J, et al. Decompressive Surgery for the Treatment of Malignant Infarction of the Middle Cerebral Artery (DESTINY): a randomized, controlled trial. Stroke. 2007;38:2518-25.

15. Vahedi K, Vicaut E, Mateo J, Kurtz A, Orabi M, Guichard J-P, et al. Sequential-design, multicenter, randomized, controlled trial of early decompressive craniectomy in malignant middle cerebral artery infarction (DECIMAL Trial). Stroke. 2007;38:2506-17.

16. Hofmeijer J, Kappelle LJ, Algra A, Amelink GJ, van Gijn J, van der Worp HB, et al. Surgical decompression for space-occupying cerebral infarction (the Hemicraniectomy After Middle Cerebral Artery infarction with Life-threatening Edema Trial [HAMLET]): a multicentre, open, randomised trial. Lancet Neurol. 2009;8:326-33.

17. Vahedi K, Hofmeijer J, Juettler E, Vicaut E, George B, Algra A, et al. Early decompressive surgery in malignant infarction of the middle cerebral artery: a pooled analysis of three randomised controlled trials. Lancet Neurol. 2007;6:215-22.

18. Yang M-H, Lin H-Y, Fu J, Roodrajeetsing G, Shi S-L, Xiao S-W. Decompressive hemicraniectomy in patients with malignant middle cerebral artery infarction: A systematic review and meta-analysis. Surgeon. 2015;13:230-40.

19. Cho D-Y, Chen T-C, Lee H-C. Ultra-early decompressive craniectomy for malignant middle cerebral artery infarction. Surg Neurol. 2003;60:227-32; discussion 232-233.

20. Kürten S, Munoz C, Beseoglu K, Fischer I, Perrin J, Steiger H-J. Decompressive hemicraniectomy for malignant middle cerebral artery infarction including patients with additional involvement of the anterior and/or posterior cerebral artery territory-outcome analysis and definition of prognostic factors. Acta Neurochir (Wien). 2018;160:83-9.

21. Cheung A, Telaghani CK, Wang J, Yang Q, Mosher TJ, Reichwein RK, et al. Neurological recovery after decompressive craniectomy for massive ischemic stroke. Neurocrit Care. 2005;3:216-23.

22. Wartenberg KE. Malignant middle cerebral artery infarction. Curr Opin Crit Care. 2012;18:152-63.

23. Pandhi A, Tsivgoulis G, Goyal N, Ishfaq MF, Male S, Boviatsis E, et al. Hemicraniectomy for Malignant Middle Cerebral Artery Syndrome: A Review of Functional Outcomes in Two High-Volume Stroke Centers. J Stroke Cerebrovasc Dis. 2018;27:2405-10.

\section{Tables}

Table 1. Pre-operative data of patients with $\mathrm{MCl}$ 


\begin{tabular}{|c|c|c|c|c|}
\hline & $\begin{array}{l}\text { DHC } \\
n=67\end{array}$ & $\begin{array}{l}\text { DHC+S } \\
n=28\end{array}$ & $\begin{array}{l}\text { Total } \\
n=95\end{array}$ & P-value \\
\hline \multicolumn{5}{|l|}{ Sex } \\
\hline Male & $37(55.2 \%)$ & $16(57.1 \%)$ & $53(55.8 \%)$ & 0.864 \\
\hline Female & $30(44.8 \%)$ & $12(42.9 \%)$ & $42(44.2 \%)$ & \\
\hline Age (mean \pm SD) & $60.64 \pm 10.68$ & $57.46 \pm 10.43$ & $59.71 \pm 10.65$ & 0.186 \\
\hline Pre-op GCS (median) & $9(5-13)$ & $8(5-12)$ & $9(5-13)$ & 0.259 \\
\hline Pre-op NIHSS (median) & $23(18-32)$ & $25(20-36)$ & $24(18-36)$ & 0.147 \\
\hline Hours from ictus to operation (mean $\pm S D$ ) & $17.41 \pm 5.60$ & $18.23 \pm 5.95$ & $17.65 \pm 5.68$ & 0.524 \\
\hline \multicolumn{5}{|l|}{ Site of infarction } \\
\hline MCA only & $46(68.7 \%)$ & $11(39.3 \%)$ & $57(60.0 \%)$ & 0.008 \\
\hline MCA+ACA and/or PCA & $21(31.3 \%)$ & $17(60.7 \%)$ & $38(40.0 \%)$ & \\
\hline \multicolumn{5}{|l|}{ Etiology of ischemia } \\
\hline Large-vessel Atherosclerosis & $22(32.8 \%)$ & $9(32.1 \%)$ & $31(32.6 \%)$ & 0.714 \\
\hline Cardioembolic & $26(38.8 \%)$ & $8(28.6 \%)$ & $34(35.8 \%)$ & \\
\hline Coagulopathy & $11(16.4 \%)$ & $6(21.4 \%)$ & $17(17.9 \%)$ & \\
\hline Other/unknown & $8(11.9 \%)$ & $5(17.9 \%)$ & $13(13.7 \%)$ & \\
\hline \multicolumn{5}{|l|}{ Preop head CT } \\
\hline Marginal swelling ${ }^{1}$ & $25(37.3 \%)$ & $5(17.9 \%)$ & $30(31.6 \%)$ & 0.007 \\
\hline Pronounced swelling ${ }^{2}$ & $34(50.7 \%)$ & $12(42.9 \%)$ & $46(48.4 \%)$ & \\
\hline Massive swelling 3 & $8(11.9 \%)$ & 11(39.3\%) & $19(20 \%)$ & \\
\hline
\end{tabular}

${ }^{1}$ Midline shift at the level of septum pellucidum $<5 \mathrm{~mm}$;

${ }^{2}$ Midline shift at the level of septum pellucidum $\geq 5 \mathrm{~mm}$ and $<10 \mathrm{~mm}$;

${ }^{3}$ Midline shift at the level of septum pellucidum $\geq 10 \mathrm{~mm}$

Abbreviations: $\mathrm{MCl}$, malignant cerebral infarction; $\mathrm{DHC}$, decompressive hemicraniectomy; $\mathrm{DHC}+\mathrm{S}$, DHC+strokectomy; SD, standard deviation; Pre-op, pre-operative; GCS, Glasgow Coma Score; NIHSS, National Institutes of Health Stroke Scale; MCA, middle cerebral artery; ACA, anterior cerebral artery; PCA, posterior cerebral artery; CT, computed tomography. 
Table 2. Observational data in ICU.

\begin{tabular}{|llll|}
\hline & $\mathrm{DHC}(\mathrm{n}=67)$ & $\mathrm{DHC}+\mathrm{S}(\mathrm{n}=28)$ & $\mathrm{P}$ \\
\hline Mannitol therapy & $50(74.6 \%)$ & $10(35.7 \%)$ & 0.000 \\
\hline Hypothermia & $31(46.3 \%)$ & $4(14.3 \%)$ & 0.003 \\
\hline Hypertonic saline & $31(46.3 \%)$ & $3(10.7 \%)$ & 0.001 \\
\hline Tracheotomy & $50(74.6 \%)$ & $22(78.6 \%)$ & 0.682 \\
\hline Acute renal failure & $6(9.0 \%)$ & $1(3.6 \%)$ & 0.670 \\
\hline Remedial surgery recommended & $16(23.9 \%)$ & 0 & 0.002 \\
\hline ICU stay (days) & $11.64 \pm 4.12$ & $9.82 \pm 2.54$ & 0.033 \\
\hline Mechanical ventilation (days) & $8.12 \pm 3.72$ & $6.75 \pm 2.63$ & 0.077 \\
\hline Mortality in ICU & $13(19.4 \%)$ & $2(7.1 \%)$ & 0.217 \\
\hline
\end{tabular}

Abbreviations: ICU, intensive care unit; DHC, decompressive hemicraniectomy, DHC+S, DHC+strokectomy.

Table 3. Characteristics of patients in the DHC group with or without malignant high ICP 


\begin{tabular}{|c|c|c|c|}
\hline & Group 1(16) & Group 2(51) & P-value \\
\hline Sex $\bigotimes M / F \otimes$ & $7 / 9$ & $32 / 19$ & 0.179 \\
\hline Age $($ mean $\pm S D)$ & $61.25 \pm 11.68$ & $60.49 \pm 10.47$ & 0.837 \\
\hline Pre-op GCS (median) & $7.5 \rrbracket 5-12 \rrbracket$ & $9(5-13)$ & 0.174 \\
\hline Pre-op NIHSS (median) & 26(19-32) & $22(18-32)$ & 0.063 \\
\hline Hours from ictus to operation (Mean $\pm S D$ ) & $17.06 \pm 5.24$ & $17.52 \pm 5.75$ & 0.778 \\
\hline Site of infarction & & & 0.004 \\
\hline MCA only & $6(37.5 \%)$ & $39(76.5 \%)$ & \\
\hline MCA+ACA and/or PCA & $10(62.5 \%)$ & $12(23.5 \%)$ & \\
\hline Preop head CT & & & 0.601 \\
\hline Marginal swelling & $6(37.5 \%)$ & $19(37.3 \%)$ & \\
\hline Pronounced swelling & $7(43.8 \%)$ & $27(52.9 \%)$ & \\
\hline Massive swelling & $3(18.8 \%)$ & $5(9.80 \%)$ & \\
\hline Mannitol therapy & $16(100 \%)$ & $35(68.6 \%)$ & 0.008 \\
\hline Hypothermia & $11(68.8 \%)$ & $20(39.2 \%)$ & 0.039 \\
\hline Hypertonic saline & $10(62.5 \%)$ & $21(41.2 \%)$ & 0.136 \\
\hline Acute renal failure & $4(25.0 \%)$ & $2(3.9 \%)$ & 0.026 \\
\hline
\end{tabular}

Abbreviations: DHC, decompressive hemicraniectomy; ICP, intracranial pressure; $\mathrm{M}$, male, F, female; SD, standard deviation; Pre-op, pre-operative; GCS, Glasgow Coma Score; NIHSS, National Institutes of Health Stroke Scale; MCA, middle cerebral artery; ACA, anterior cerebral artery; PCA, posterior cerebral artery; CT, computed tomography.

Table 4. Midline shift calculations before and 1 day after surgery

\begin{tabular}{|llll|}
\hline & $\mathrm{DHC}$ & $\mathrm{DHC}+\mathrm{S}$ & P-value \\
\hline Pre-op & $6.48 \pm 2.22$ & $8.07 \pm 2.14$ & 0.002 \\
\hline Post-op & $6.81 \pm 2.09$ & $4.46 \pm 1.00$ & 0.000 \\
\hline
\end{tabular}

Abbreviations: DHC, decompressive hemicraniectomy; $\mathrm{DHC}+\mathrm{S}$, DHC+strokectomy; Pre-op, pre-operative; Post-op, post-operative.

Table 5. Functional outcomes and mortality at 6 months. 


\begin{tabular}{|llll|}
\hline Outcome & DHC (67) & DHC+PS (28) & P-value \\
\hline Good (mRS 0-3) & $20(29.9 \%)$ & $6(21.4 \%)$ & 0.401 \\
\hline Poor (mRS 4-5) & $30(44.8 \%)$ & $20(71.4 \%)$ & 0.018 \\
\hline Mortality (mRS=6) & $17(25.4 \%)$ & $2(7.1 \%)$ & 0.043 \\
\hline
\end{tabular}

Abbreviations: DHC, decompressive hemicraniectomy; DHC+S, DHC+strokectomy; mRS, modified Rankin Scale.

\section{Figures}

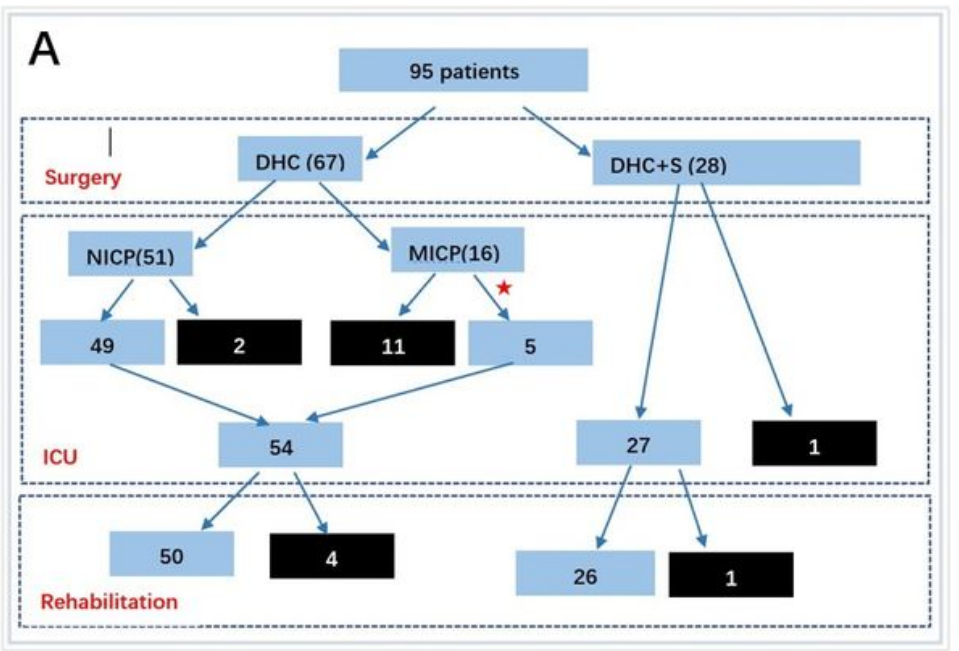

\section{B}
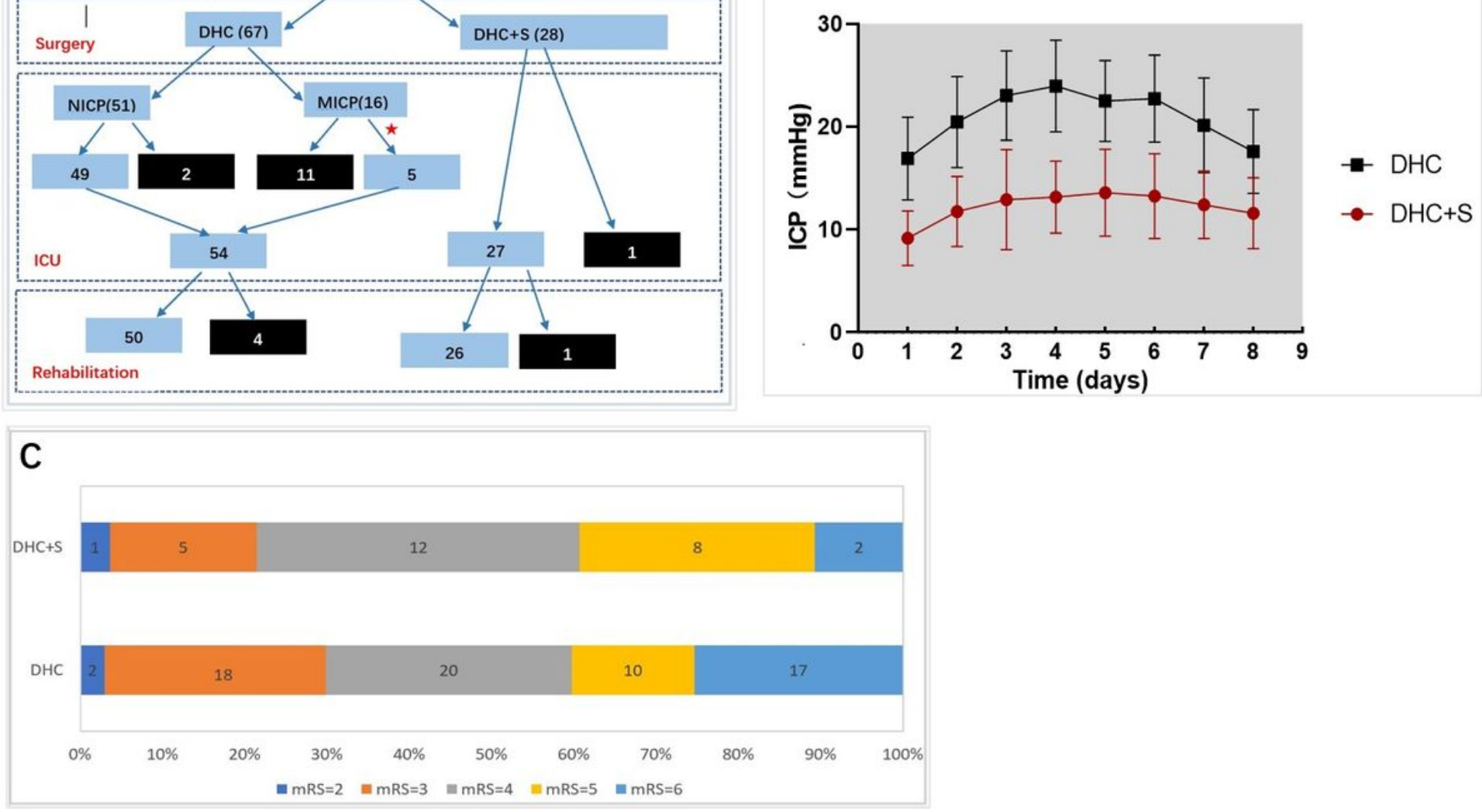

\section{Figure 1}

A: Overview of the included patients. B: The comparison of ICP curves in DHC group and DHC+S group. Note that DHC+S group had much lower ICP compared to DHC group after surgery. C: Bar graph showing the distribution of six-month patient functional outcomes as determined by mRS. The numbers within each box denote the number of patients. 